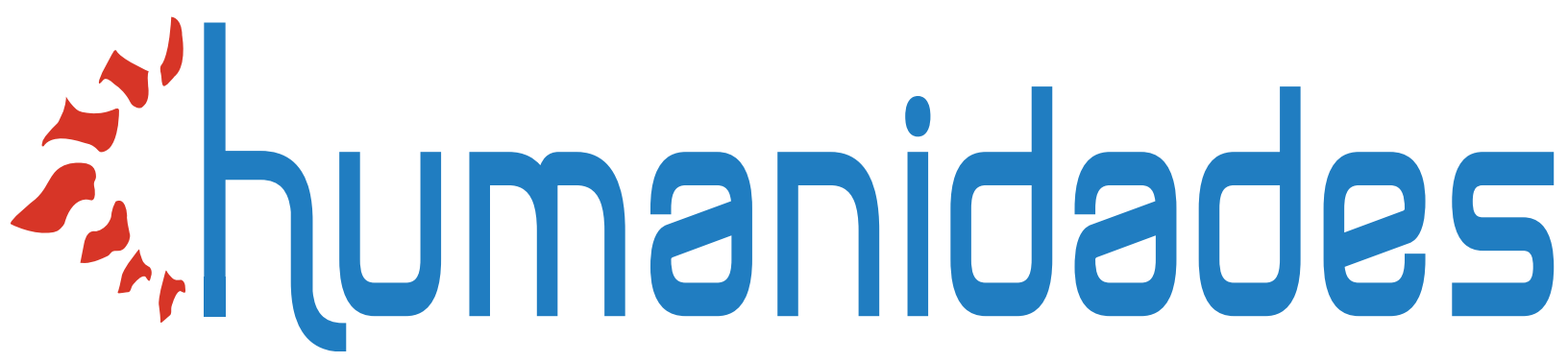

\title{
Bruce Chatwin: Utz, su novela sobre el mundo del arte
}

\author{
Isabel López Hernández
}

DOI: https://doi.org//0.155 I7/h.v10i2.4I806 https://revistas.ucr.ac.cr/index.php/humanidades/index 


\section{"humanidades}

Revista Humanidades

ISSN: 2215-3934

humanidades@ucr.ac.cr

Universidad de Costa Rica

Costa Rica

\section{Bruce Chatwin: Utz, su novela sobre el mundo del arte}

López Hernández, Dra. Isabel

Bruce Chatwin: Utz, su novela sobre el mundo del arte

Revista Humanidades, vol. 10, núm. 2, 2020

Universidad de Costa Rica, Costa Rica

Disponible en: http://www.redalyc.org/articulo.oa?id=498062469012

DOI: https://doi.org/10.15517/h.v10i2.41806

Esta obra está bajo una Licencia Creative Commons Atribución-NoComercial-SinDerivar 3.0 Internacional. 
Desde el arte, la literatura y la comunicación

\title{
Bruce Chatwin: Utz, su novela sobre el mundo del arte
}

\author{
Bruce Chatwin: Utz, His Novel About the Art-World
}

Dra. Isabel López Hernández

Universidad Politécnica de Madrid, España

isabel.lopez@upm.es

DOI: https://doi.org/10.15517/h.v10i2.41806

Redalyc: http://www.redalyc.org/articulo.oa?

iD http://orcid.org/0000-0002-3134-8187

$\mathrm{id}=498062469012$

Recepción: 23 Marzo 2020

Aprobación: 28 Abril 2020

\section{Resumen:}

El escritor británico Bruce Chatwin (1940-1989) convierte a un coleccionista compulsivo en eje central de su novela Utz. A través de su protagonista, reflexiona acerca del mundo del arte, rememorando su pasado cuando trabajaba en la casa de subastas Sotheby's. Su experiencia allí le proporcionó unos conocimientos y una mirada que determinarían su vida, aprendió a estudiar una obra de arte, a describirla en pocas palabras y valorar su precio en el mercado. Todo ello se percibe en su estilo narrativo, donde persigue la precisión de las descripciones típicas de los catalogadores. Desarrolló un estilo propio. Además, adquirió una red de contactos trascendental alrededor del mundo. Fijó su interés en personalidades peculiares que desembocaron en la creación de Utz, personificación de ese entramado de marchantes y coleccionistas que le facilitó su puesto de director en el departamento de impresionistas y arte moderno. Al mismo tiempo, su época en Sotheby's despertó en él la eterna disyuntiva entre la posesión, la búsqueda de la inmortalidad y la creación artística. El presente artículo examina cómo el autor aborda todos estos temas en la novela.

Palabras Clave: Porcelana, Colección de arte, Museo.

\section{Abstract:}

British writer Bruce Chatwin (1940-1989) makes a compulsive collector the central axis of his novel Utz. Through that character, he reflects on the art world remembering his work at the auction house Sotheby's. His experience there gave him the knowledge and look that would condition his life. He learnt to analyze a work of art, to describe it in just a few words and to value it in the market. All of it can be noticed in his writing style, in which he pursues the kind of the precision observed in cataloguers. He developed his own technique. Besides, he acquired a strong contact network around the world. He put his eyes on peculiar personalities that led to the creation of Utz, personification of all those art dealers and collectors that he met during his time as the head of the firm's Impressionist and Modern Art department. Moreover, his work at Sotheby's awakened his eternal dilemma between possession, search for immortality and creation. This article examines how the author treats the above topics in his novel. KEYWORDS: Porcelain, Art collection, Museum.

\section{INTRODUCCIÓN}

Para Bruce Chatwin lo opuesto al nómada era el coleccionista (Clapp, 1997). Sobre este tema versa su última novela, Utz. El escritor, crea un personaje, el aristócrata checo Kaspar Utz, coleccionista compulsivo de figuritas de porcelana Meissen, a partir de él elabora un cuento de misterio, una evocación de Europa Oriental y además explora la naturaleza de la pasión por acumular. Temáticamente $U t z$ brilla por ser uno de los libros más ricos de su bibliografía, a pesar de su brevedad. Chatwin analiza en él muchas de las inquietudes ya tratadas en sus obras anteriores, profundiza en sus obsesiones y añade nuevos argumentos. Consigue, además, conectar todos los ingredientes magistralmente. Acomete un estudio sobre la psicopatología del coleccionista compulsivo, analiza la leyenda del gólem y esboza la historia de Checoslovaquia en apenas 140 páginas. El autor provecha también para recapacitar sobre lo efímero y lo eterno (Clapp, 1997).

El lector se enfrenta a una obra donde se percibe a un Chatwin que desea volcar su sabiduría, este hecho se explica al conocer que, durante la redacción de aquella, el autor sabía que su fin estaba cerca. Sentía que el SIDA iba deteriorando su cuerpo (Shakespeare, 1999). Las meditaciones que plasma acerca de la relación del 
arte y la muerte deben entenderse como una última reflexión sobre su forma de entender la vida (Meanor, 1996). Aprovecha también para despedirse de su esposa Elizabeth Chandler, a la que había conocido en Sotheby's. Introduce en la novela un final sentimental como tributo al compromiso nada convencional que había establecido con ella. Se inspira en ella, además, para construir a uno de los personajes, Marta. Irónicamente, Utz se alza como una de las obras más humorísticas de Chatwin (Clapp, 1997).

El texto constituye una oda a la mutabilidad de las cosas e invita a un profundo examen sobre la permanencia de los objetos frente a la volatilidad de la vida. Sin embargo, la seriedad temática cuenta con una cualidad antes desconocida en el autor: su capacidad para reírse de sí mismo y de la vacuidad del comercio del arte. Como su amigo, el escritor Salman Rushdie manifestó, $U t z$ indicaba que Chatwin comenzaba realmente una nueva fase en su carrera como escritor una vez que había volcado en Los trazos de la canción, su odisea australiana, sus obsesiones (Rushdie, 1992).

\section{FuENTES DE INSPIRACIÓN PARA LA CREACIÓN DE LA NOVELA}

Para perfilar la historia de Utz, el personaje central de la novela, el autor se remontó a su época en Sotheby's, la casa de subastas, y su posterior estancia en Edimburgo, donde estudió arqueología. Ambos mundos le tendieron una misma trampa, como él manifestaba, ya que vivía muy pendiente de los objetos (Ignatieff, 1987). Fue entonces cuando comenzó a interesarse por la vida de los nómadas, aquellos que viajaban ligeros de equipaje, sin cargas innecesarias. Poco a poco se fue desprendiendo de objetos que había amontonado, sobre todo, de los más recargados. Conservó solo aquellos que parecían más simples o significativos. Pero siempre lucharía contra esa parte de él que inevitablemente le impelía a conservar y a coleccionar. Una de las razones por las que decidió escribir esta novela guarda relación con este conflicto (Clapp, 1997).

Chatwin dio vida a Utz basándose en varios personajes que había conocido durante su etapa en Sotheby's: Dr. Rudolph Just, Konstantin Melnikov, George Costakis y George Ortiz. "El coleccionista era otra de las importantes categorías de Bruce: lo opuesto al nómada; la persona que -declaraba él de cuando en cuandoera importante dejar de ser" (Clapp, 1997, p. 273). Mezcló los encuentros que mantuvo con todos ellos con su experiencia atesorada durante su vida profesional en la casa de subastas. Aderezó el conjunto con aspectos personales obtenidos de su biografía que encajaban en la idea de libro que deseaba escribir. En concreto, la novela nació durante una visita de Chatwin a Checoslovaquia. Allí conoció a un amante de la porcelana, el Dr. Rudolph Just, que le serviría como uno de los modelos a la hora de dar forma al protagonista de su última obra.

Muchos aspectos de la vida del coleccionista se repiten en la novela, como, por ejemplo, la existencia de una colección y su inexplicable desaparición. También se enfrentó con las autoridades checas y se casó con su ama de llaves. Su encuentro con él fue muy breve, pero una vieja amistad de Sotheby's que trabajaba en el departamento de porcelana le proporcionó los datos necesarios para dibujar un esquema de la personalidad de su personaje. Chatwin introdujo una serie de modificaciones para proteger al hijo del Utz real, que residía en Praga y podría saber del paradero de la colección. Chatwin alteró las circunstancias para darle brillo. El tesoro del Utz real era muy variado, no como el del personaje de la novela, que consistía solo en figurillas Meissen. De esta manera, consigue retratar a un coleccionista más obsesivo, de igual menera, también ensambló otros elementos que infundieron un aspecto más interesante a la historia.

Algunos objetos que Chatwin describe en la novela reproducen modelos vendidos en su época de Sotheby's que no guardan ninguna analogía con el Utz real, por ejemplo, el arlequín que sostiene una jarra de cerveza sentado sobre el tronco del árbol, reflejado en la novela, fue una figura vendida en la casa de subastas durante su época allí. Además, Chatwin elige elementos artísticos con unas características que representan lo opuesto a lo que él consideraba bello, acentuando así su distanciamiento de la profesión que por unos años llenó su vida. A pesar de no recurrir a su infancia como fuente fundamental de la novela, sí acudió una vez más a ella con el fin de rescatar datos concretos que pudiera incluir. Así, el diminuto apartamento de Utz es una versión 
de la casa donde pasó gran parte de su niñez en Birmingham (en el número 198 de West Heath Road) y se apoyó en la vitrina de su abuela para retratar los estantes con espejos del protagonista de la novela (Clapp, 1997).

El arquitecto ruso Konstantin Melnikov, al que visitó en Moscú en 1973, pasó también a formar parte de la idiosincrasia de su personaje. Chatwin quedó fascinado por la casa-cárcel donde el artista vivió durante cuarenta años recluido por las autoridades soviéticas. Calificó la morada de maravilla arquitectónica del siglo XX (Chatwin, 2002). La soledad de Melnikov recuerda a la de Utz, quien también eligió su vivienda en Praga para convertirla en una prisión y vivir rodeado de sus porcelanas.

Otro personaje en el que se basó para dar vida a su protagonista fue George Costakis, coleccionista privado soviético al que entrevistó en la misma época. Costakis transformó su domicilio en un museo de arte futurista y las autoridades le permitieron que la conservara mientras viviera. Tras su muerte, esta pasó a manos soviéticas. El paralelismo con la historia de Utz resulta evidente:

Parece que las autoridades comunistas — siempre dispuestas a asumir una apariencia de legalidad- permitieron que Utz conservara sus porcelanas con la condición de que dejara fotografiar y numerar cada pieza. También se convino - aunque nunca por escrito- que, después de su muerte, los museos del Estado se quedarían con todo (Chatwin, 1989, p.27).

Otro personaje que pudo influir en la elaboración del Utz imaginario se trata de su amigo, también famoso por su colección de arte, George Ortiz, que se consideraba a sí mismo una víctima de su propia pasión. Compartía con el escritor británico la opinión de que las obras de arte arrancan un trozo de vida a los que las coleccionan. La admiración de Ortiz por el gusto de Chatwin constituía, según él, la base de su amistad. Percibían las piezas artísticas de la misma manera. Chatwin, sin embargo, se liberó de los objetos consiguiendo que estos no significaran nada para él. Solía bromear con Ortiz acerca del personaje que había creado con Utz diciendo que se basó en él para elaborar la novela. Ortiz odiaba las figuritas Meissen y Chatwin lo sabía. De ahí la broma (Shakespeare, 1999).

El propio autor se escondió detrás de Utz. La intriga en cuanto a la clase de relación entre el héroe de la novela y Marta, su ama de llaves, se asemeja al propio matrimonio de Chatwin con Elisabeth. Volcó mucho de sí mismo en la relación de los protagonistas. La vida sexual del coleccionista constituye una de las sorpresas de la novela, como también lo fue en la vida del autor. Utz tiene colgada en su apartamento una bata de seda de color melocotón con tonos rosas y un cuello de plumas de avestruz haciendo juego. Cuando el narrador descubre esta pertenencia, se desencadena en su imaginación un torbellino de ideas. Chatwin describe con comicidad cómo el narrador comienza a observar a Utz bajo otra perspectiva ahora que ha descubierto que puede tratarse de un travestido. Sospecha que un recipiente de talco puede contener en realidad polvos faciales y que bajo los volantes del tocador esconde quizá una peluca. Todo en la morada del protagonista sugiere un lado femenino muy acentuado: "Las cortinas rosadas con volantes y el edredón rosado de raso — que, en ambos casos, habían visto días mejores - conferían a la habitación una atmósfera de femineidad anticuada, un poco basta" (Chatwin, 1989, p. 96). Finalmente, se descubre que la existencia de esos toques se debe a que Utz y Marta comparten alcoba como marido y mujer. El autor juega con los malentendidos. Sorprende así al lector y lo vuelve a hacer cuando insinúa que más que un matrimonio de conveniencia se trataba de una unión nacida del amor.

El vínculo que Chatwin mantenía con su esposa sorprendía tanto a amigos como a familiares. Algunos ni siquiera llegaron a conocer a Elizabeth. A otros, como al escritor Paul Theroux, les gustaba el misterioso papel de su compañera. El simple hecho de que estuviera casado ya era sorprendente. Cuando se publicó la novela, en septiembre de 1988, el escritor dependía físicamente de Elizabeth. Hacia el final de sus días se produjo una reconciliación entre ambos que recuerda a esa declaración tardía de amor que se observa en Utz. Al igual que su creador, Utz también huye en busca de una parcela lo más alejada posible de su esposa. Sus viajes a Vichy representan en cierto modo esa escapada lejos del hogar, un modo de liberarse: "Hacia abril, también experimentaba una claustrofobia aguda, producto de haber pasado los meses de invierno muy 
cerca de Marta, que no cesaba de adorarlo” (Chatwin, 1989, p. 84). Pero el regreso a casa junto a Marta era siempre inevitable. Algunos párrafos donde se detalla la relación entre Marta y Utz parecen autobiográficos: "Le remordía la conciencia porque la había abandonado: a la pobrecilla que lo adoraba; que daría su vida por él, con su corazón apasionado que palpitaba por él, y sólo por él” (Chatwin, 1989, p. 79). Elizabeth también comparte con Marta la capacidad de sufrimiento y la paciencia. Ambas mujeres esperan estoicamente a que su amante regrese y no reprochan nada (Clapp, 1997).

\section{UTZ: LA REFLEXIÓN DE CHATWIN SOBRE SU PASADO EN EL MUNDO DEL ARTE}

Chatwin utilizó a $U t z$ para meditar acerca de su eterna disyuntiva sobre el mundo del arte. El tema le obsesionaba y nunca pudo desprenderse de este plenamente. Durante un viaje por Patagonia, lejos de cualquier museo o galería, escribió en su cuaderno de notas que resultaba fascinante cómo el ser humano utiliza los objetos como sustituto de otro tipo de relaciones (Shakespeare, 1999). Chatwin debía mucho a ese universo. Tuvieron que pasar más de veinte años desde que dejara Sotheby's para que decidiese escribir sobre él. Esa época dejó una huella indeleble en su obra, no solo temáticamente, sino también formalmente (Clapp, 1997).

El método de los catalogadores aprendido en la casa de subastas puede percibirse en su manera de estructurar los párrafos y tramas. Como hizo durante sus años en Sotheby's, Chatwin reúne, tanto en $U t z$ como en el resto de sus obras, una gran cantidad de detalles, busca fuentes y enlaza historias con la minuciosidad del oficio preciosista que un día ejerció. A pesar de la brevedad de su novela, consigue mezclar temas complejos y recrearse en reflexiones filosóficas profundas, aunque persiguiendo siempre la objetividad y el origen. Sus referencias a personajes históricos son constantes, desde Kepler hasta Charlie Chaplin, pasando por Marco Polo, Arcimboldo y Marx, por nombrar solo algunos. Una muestra de la erudición presente en otras obras que, sin duda, tuvo su germen en Sotheby's (Chatwin, 2002).

Chatwin pasó de coleccionar objetos a catalogar experiencias una vez que decidió abandonar su puesto en la casa de subastas. Intentó alejarse de ese mundo, pero su obsesión regresó en forma de pasión por reunir material, investigar e idear historias. Utz confirmó que su duelo con el coleccionismo, al que reprochaba tanto, pero del que no podía escapar, no había llegado a su fin (Gnoli, 2002). Chatwin nunca se liberó de sus propios fantasmas estéticos y nadaba siempre en un mar de contradicciones, ya que su propia filosofía de vida implicaba la renuncia a toda posesión. La paradoja era la quintaesencia de su personalidad. Por esta razón, le interesaban los comunistas ricos, la idea de una luz oscura o los artistas sensibles que eran seres humanos brutales (Clapp, 1997). El poeta Arthur Rimbaud y Chatwin "obraron sobre el confín de la mercancía, sobre aquello que representa su límite. Comerciar en armas, o acaso en esclavos, representó para Rimbaud la manera de liberarse de la idea de que las mercancías son inocuas, que el valor es inocente" (Gnoli, 2002, p. 49). Chatwin trató de forzar la idea de mercancía abordándola por el lado del coleccionismo y llegó a la conclusión de que constituía la mayor insensatez del poder mercantil. El objeto coleccionado encarnaba para el escritor británico en parte un obsequio y en parte una mercancía. De aquí nacía la obsesión por el coleccionismo y, en consecuencia, su ambigüedad (Gnoli, 2002).

El autor confesó que nunca había dado la espalda al mundo del arte, simplemente aprendió a observarlo bajo una perspectiva diferente (Gnoli, 2002). Con el paso de los años, consiguió, incluso, utilizarlo de una forma imprevisible, como lo demuestra $U t z$. Lo que aprendió fundamentalmente de aquella época se resumía en que el ambiente reinante en Sotheby's encerraba una terrible capacidad de destrucción. En su caso se tradujo en un progresivo desinterés en el proceso de creación de una colección. En sus peores momentos imaginaba que esta actividad guardaba más relación con la muerte que con la vida. Esta idea, además, se confirmó más tarde una vez que decidió iniciar sus estudios universitarios en Edimburgo. Allí comprendió que la arqueología, concretamente, se alejaba de la idea vital que dominaba su filosofía personal, se desviaba de la creencia de que la verdadera naturaleza residía en el seno del camino. 
Un buen día comprendió que el mundo del arte no le infundía alegría, puesto que representaba la rigidez y lo previsible (Chatwin, 2010). La extraña enfermedad que le diagnosticaron cuando prestaba sus servicios en Sotheby's (sufrió una ceguera psicosomática) le liberó, en cierto modo, de unos sentimientos que se habían acumulado en él durante sus años en la casa de subastas. Funcionaron como una vía de escape a su desasosiego. Su dolencia era la consecuencia lógica de haber permanecido en la oscuridad. El mundo se tiñó de colores apagados, hasta fundirse en una opacidad completa. Su afección ocular supuso la respuesta a ese sentimiento de insatisfacción que le atosigaba. El médico le sugirió que sustituyera la visión de cerca a la que estaba comprometido en su profesión, donde el horizonte era siempre un cuadro, por paisajes abiertos. En aquel momento, a Chatwin le pareció una burla del destino (Chatwin, 1988). El artista era famoso por ese ojo infalible capaz de detectar falsificaciones con tan solo un simple vistazo. La curación de su mal implicaba alejarse de su don y, por lo tanto, del éxito. No obstante, gracias a este consejo, Chatwin consiguió dar el salto desde el coleccionismo a lo más alejado que pudo encontrar: el nomadismo. Utz supuso el regreso a esa etapa que había quedado a su espalda.

Cuando Chatwin trata el tema del coleccionismo en la novela está describiendo su propio trayecto vital en torno a esa pasión. En concreto, la afición de Utz por el coleccionismo aflora al enamorarse de una figura Meissen de arlequín encerrada en una vitrina en casa de su abuela. Utz dijo, con la naturalidad de un niño: "Quiero eso", pero se le negó (Chatwin, 1989). Tras la muerte de su abuela este objeto llegará a sus manos y mientras acaricie sus contornos comprenderá que el resto de su vida lo dedicará a la colección de ese tipo de porcelanas o, como él lo llamaba, al rescate de esas figuritas de la fábrica Meissen. A partir del momento en que sus dedos acarician el esmalte del arlequín aborrecerá cualquier barrera que le impida esa unión. Esta constituye la razón de su odio a los museos. Defiende las colecciones privadas y cree que cada cincuenta años los museos deberían abrirse para permitir que la gente los saquease. De esta manera, las propiedades privadas volverían a la circulación y el arte de recopilar permanecería vivo siempre. Un objeto en una vitrina es para Utz como un animal en un zoo. Las obras de arte agonizan en los museos, mientras que el coleccionista privado devuelve el derecho al contacto humano. Le compara con un niño que intenta acariciar aquello que nombra, como él mismo hizo de pequeño. El primero devuelve al objeto su viveza, la que el creador de la obra de arte puso en ella. Se suma a las filas enemigas de aquel que niega ese contacto. Al tocar la pieza, la pasión del coleccionista privado restaura la armonía existente entre la creación y el creador. Le devuelve la vida que el artista le otorgó. Esta idea del museo como prisión del objeto se corresponde con la percepción del propio Chatwin (Meanor, 1996).

\section{UTZ: LA HISTORIA DEL GÓLEM, LA CREACIÓN Y LA BÚSQUEDA DE LA INMORTALIDAD}

Chatwin dedica mucho espacio en la novela a disertar acerca del acto de la creación y su significado para el artista. Esta cuestión se articula a través de la narración de la historia del gólem, ya que la existencia de las figuras de Meissen se relaciona íntimamente con el acto creativo. El artista y su obra poseen la capacidad de transformar el mundo. El ingrediente básico del arte reside en ese poder de cambio. En este sentido, cualquier obra de arte tiene la misma facultad de metamorfosis que la piedra filosofal. Para Utz, las porcelanas equivalen a seres vivos con esa capacidad de mutación. La existencia del gólem, por tanto, se relaciona con inmortalidad, tal y como ocurre con la posesión de las figuritas de porcelana. Con el fin de explicar esta idea, en primer lugar, Chatwin desgrana la vida del famoso rabino Loew, líder de los judíos en Praga en el siglo XVI. El rabino creó, con el barro del río Vltava, a Yossel el gólem, un hombre artificial de arcilla. Establece así una clara relación con el nacimiento del primer ser humano. Si Yossel utilizó barro del río para modelar a su ser, Dios usó arcilla para crear a Adán. Dios se erigía, de este modo, en el primer escultor y Adán en la primera escultura de cerámica: "El mismo padre Adán había sido gólem, una masa inerte de arcilla tan extensa que cubría los extremos de la Tierra: esto es, hasta que Iahvé lo redujo a la escala humana e insufló en su boca la facultad del habla" (Chatwin, 1989, p. 43). 
Utz añade otra serie de historias a esta de la creación del primer hombre. Una de sus favoritas contaba que a Jesucristo le gustaba moldear pájaros de arcilla. Tras pronunciar la fórmula sagrada, estos iniciaban su canto y adquirirían el don de volar. Con esta narración, Utz identifica la actividad del escultor de porcelanas con el creador. El artista es un Dios capaz de contagiar vida a sus creaciones. Este discurso sugiere que Utz cree que sus porcelanas albergan vida, pero cuando el narrador le pregunta abiertamente sobre ello sencillamente contesta: "Están vivas y están muertas. Pero si estuvieran vivas tendrían que morir. ¿No es verdad?” (Chatwin, 1989, p. 43). La respuesta plantea un enigma, aunque también insinúa que las invenciones artísticas superan al maestro que las alumbra. El arte de crear va más allá de las reglas humanas. Además, si sus figuras estuvieran vivas, significaría que tenderían a la desaparición. Nada más erróneo. No son terrenales, sino divinas (Murray, 1993).

Otra de las historias de Utz alude al momento en que el gólem pasa a ser más fuerte que su demiurgo y lo destruye. Esta es la relación que él sostiene con sus figuras que, en cierto modo, controlan su existencia. Su colección le mantiene prisionero. El gólem, en este caso, se convierte en una especie de Frankenstein. Si no se le somete a control, se transforma en un monstruo, dado que anhela parecerse al Adán cósmico. Aspira a dominar el mundo. Su magnitud carece de límites, por lo que resulta muy peligroso (Meanor, 1996).

En el punto culminante de la discusión acerca del gólem, Utz afirma que sus creadores eran alquimistas conocedores de los secretos arcanos. No obstante, idear una figura de aspecto humano significaba transgredir la Ley Santa. Para los judíos del siglo XVI, un icono de apariencia humana era una blasfemia que suplicaba su autodestrucción. La idolatría se consideraba un pecado. Por tanto, cuando el narrador, es decir, Chatwin, relaciona el arte de crear un gólem con el de coleccionar, expresa que reunir obras de arte podría juzgarse de fetichismo o, lo que es lo mismo, de pecado. Si el gólem representaba, por su propia existencia, una advertencia contra la idolatría e imploraba su eliminación, las figuras de Utz, indudablemente, también exigían su muerte. El narrador plantea a Utz esta cuestión problemática, aunque el protagonista no responderá a su pregunta hasta el final del libro cuando se produzca el desenlace de la trama. De este modo, consigue mantener el misterio hasta la conclusión.

Lo que Chatwin intenta transmitir con el planteamiento de estas preguntas es su visión del coleccionismo, una actividad que relacionaba con la muerte después de haber trabajado en Sotheby's. En una ocasión manifestó que el ambiente del mundo del arte le recordaba a la morgue y que cada vez que la gente alababa esas cosas preciosas que pasaban por sus manos, él se las miraba sintiéndose como Lady Macbeth (Meanor, 1996). Este aspecto mortuorio queda reflejado en la novela mediante las descripciones sobre el mausoleo donde Utz guarda sus figuritas: "El olor me resultó conocido: el tufo rancio donde se guardan obras de arte y donde consideran peligroso quitar el polvo" (Chatwin, 1989, p. 47). No solo la vitrina de Utz desprende esta idea de muerte, sino también el olor de su casa, un aroma penetrante, rancio, donde al polvo se le considera un componente benigno, transmite un ambiente opresivo donde la vida no penetra. El apartamento del protagonista simboliza la relación entre la idea de la acumulación de objetos y la visión de la vida que esta pasión implicaba para Chatwin. En la casa reina la misma atmósfera lúgubre que en Sotheby's. Cuando el narrador entra en la habitación donde yace el conjunto de porcelanas acumulado, penetra en otra realidad donde gobierna la mente del coleccionista compulsivo.

La costumbre de coleccionar fue en un tiempo catalogada bajo el nombre de Porzellankrankheit (enfermedad de la porcelana). Tan fuerte era su influjo que llegó a deformar la visión de soberanos como Augusto el Fuerte y la de sus ministros. Estos llegaron a confundir los intereses político-económicos de un país con las pasiones desatadas por este ardor sin límites (Meanor, 1996). El narrador se siente fascinado ante el poder que esta ocupación puede transmitir. La atmósfera rancia y opresiva que impregna el domicilio de Utz le transporta a otra dimensión, más cercana a la muerte que a la vida. Pero no se trata solo del olor. La forma en la que las figuritas están dispuestas, con un espejo que duplica su número, contribuye a aumentar la sensación de irrealidad que parece apoderarse de la mente del coleccionista: "Los estantes tenían espejos en la parte posterior, de modo que experimentabas la ilusión de entrar en una doble hilera de salones 
refulgentes, un palacio de ensueño multiplicado hasta el infinito, donde las formas humanas revoloteaban como sombras insustanciales" (Chatwin, 1989, p. 49). La disposición de los objetos parece indicar que van a permanecer así eternamente. Por otra parte, ese escenario de ensueño en el que habitan las figuritas de porcelana se contrapone a la temporalidad a la que están sujetos los dos personajes. Utz y el narrador observan las estanterías con la sensación de que ellos desaparecerán mientras que ese palacio mítico permanecerá para siempre en esa misma posición. El rostro del protagonista se le antoja al narrador que es de cera derretida. Mientras que las figuras de porcelana parecen inmutables, el rostro de Utz envejece ante sus ojos. La escena encierra en sí una alusión a la estrecha relación existente entre el arte de coleccionar y la búsqueda de la inmortalidad.

Utz comenta que la porcelana china se consideraba una sustancia de la que se podía obtener la juventud eterna. Bottger inventó esta materia prima en el siglo XVII, a partir de unos experimentos de alquimista que finalmente le condujeron a su descubrimiento. La porcelana adquirió desde ese momento unos tintes mágicos y llegó a considerarse el antídoto de la vejez (Meanor, 1996). Arrancando de esta idea, Chatwin llega a la conclusión de que las cosas son más fuertes que las personas. Los objetos perduran, mientras que los seres vivos desaparecen inevitablemente víctimas de su fugacidad: "Los objetos son el espejo inmutable en el que vemos cómo nos desintegramos. Nada es más envejecedor que una colección de obras de arte" (Chatwin, 1989, p. 107).

Esta idea de que las mercancías sobreviven a las personas recuerda a Keats y su poema "Oda a una Urna Griega”. El poeta, describiendo la decoración de la urna, resalta la eternidad de la escena reproducida. Los personajes allí tallados perdurarán para siempre en ella, en la misma postura. El artista ha conseguido detener ese instante perpetuamente. Meanor recuerda que Chatwin introduce en este momento de la novela un tema clásico de la literatura resumido en el epigrama: Vita brevis ars longus. Añade que se puede considerar el desenlace emocional y espiritual de la novela., el cual encierra en sí el mensaje primero del libro (Meanor, 1996). Utz muestra al narrador, en este momento, todos y cada uno de los personajes de la Comedia del Arte. Las figuras han sido captadas en un instante concreto y permanecerán así indefinidamente. Scaramouche rasgueará su guitarra, mientras el Capitán dará muestras de su fanfarronería pueril de manera persistente. Los espaguetis permanecerían para siempre suspendidos en las fosas nasales de Pulchinella y Colombina nunca se desenamorará de Arlequín:

Y Arlequín... El Arlequín... el superimprovisador, el tramoyista, maestro de la versatilidad... siempre se pavonearía con su plumaje variopinto, sonreiría a través de su máscara anaranjada, entraría de puntillas en las alcobas, vendería pañales para los hijos del Gran Eunuco, bailaría sobre el filo de la catástrofe... ¡El señor Camaleón en persona! (Chatwin, 1989, p. 108).

Estas palabras dedicadas al arlequín describen simultáneamente a Utz. Cuando Utz y el narrador observan las estanterías cobran conciencia de su mortalidad. Ellos personifican simples héroes etéreos frente a la eternidad de las porcelanas. Para Utz, "la porcelana china, continuó, era una de esas sustancias legendarias, como el cuerno del unicornio o el oro alquímico, de las cuales los hombres esperaban poder beber el contenido de la Fuente de Juvencia" (Chatwin, 1989, p. 98). Chatwin, además, está explicando la íntima conexión que enlaza a la alquimia y al desarrollo de la creatividad. Para Utz, este arte quimérico constituye un ejercicio místico. Tanto la búsqueda del oro como la persecución de la porcelana confluían en un mismo objetivo: hallar la sustancia que permitiera alcanzar la inmortalidad. Utz admite que había emprendido sus estudios acerca de la alquimia con el propósito de convertir su pasión en ese ejercicio místico. De este modo, podrían apoderarse de su colección, pero él continuaría poseyéndola igualmente en un plano superior inalcanzable. Sus lecturas de Jung, Goethe y el Dr. Dee, entre otros eruditos, le ayudaron a avanzar en este camino del misticismo. Chatwin está afirmando con toda esta disertación que la nigromancia no debe relegarse al campo del ocultismo, sino que ayudan a comprender más profundamente la historia de la ciencia, la religión y la psicología (Meanor, 1996). Durante la disertación de Utz se plantea la duda de si las porcelanas viven o no. El hecho de que la imaginación del siglo XVIII considerara esta sustancia un antídoto frente al deterioro 
hace que Utz la conciba como la manera de alargar su existencia. Aumentar la adquisición de estos poderosos talismanes, equivale a dar un paso más hacia la inmortalidad. Que estén vivas o no carece de importancia para el protagonista.

\section{ConClusión}

Utz es la novela donde Chatwin construye un personaje antinómada, un coleccionista compulsivo. Elabora, en este sentido, una síntesis de aquel ajetreo de antigüedades vivido durante aquella época en Sotheby's. Analiza, además, la estrecha relación entre la posesión, la búsqueda de la inmortalidad y el acto creativo. Se puede afirmar que es su obra más rica temáticamente. Chatwin utilizó a $U t z$ para reflexionar acerca de su eterna disyuntiva sobre el mundo del arte. El tema le obsesionaba y nunca pudo desprenderse de él plenamente. Durante un viaje por Patagonia, lejos de cualquier museo o galería, escribió en su cuaderno de notas (como se citó en Shakespeare, 1999): "Es fascinante cómo el hombre utiliza los objetos como sustituto de otro tipo de relaciones". Chatwin debía mucho a ese mundo, tuvieron que pasar más de veinte años desde que dejara Sotheby's para que decidiese escribir sobre él. Esa época dejó una huella indeleble en su obra, no solo en cuanto a la temática, sino también en cuanto a la forma. El método de los catalogadores aprendido en la casa de subastas puede percibirse en su manera de estructurar los párrafos y tramas. Como hizo durante sus años en Sotheby's, Chatwin reúne, tanto en Utz como en el resto de sus obras, una gran cantidad de detalles, busca fuentes y enlaza historias con la minuciosidad del oficio preciosista que un día ejerció.

A pesar de la brevedad de su novela, consigue mezclar temas complejos y recrearse en cavilaciones filosóficas profundas, aunque persiguiendo siempre la objetividad y el origen. La novela constituye una muestra de su erudición que, sin duda, tuvo su germen en Sotheby's. Por otra parte, sabía que su fin no estaba lejos. Deseaba volcar el mayor número posible de meditaciones intelectuales y vitales que pudiera en un libro de apenas 140 páginas. La nota triste es que, aunque el libro anunciaba una nueva etapa como escritor de ficción, dejó a los lectores sin saber qué otros caminos literarios habría transitado en un futuro.

\section{REFERENCIAS}

Chatwin, B. (1988). Los trazos de la canción. Barcelona: Ediciones Península.

Chatwin, B. (1989). Utz. London: Penguin Books.

Chatwin, B. (2002). Qué hago yo aqui. Barcelona: El Aleph.

Chatwin, B. (2010). Bajo el sol. Las cartas de Bruce Chatwin. México: Editorial Sexto Piso.

Clapp, S. (1997). Con Bruce Chatwin. Barcelona: Muchnik Editores.

Gnoli, A. (2002). Bruce Chatwin: la nostalgia del espacio. Barcelona: Editorial Seix Barral.

Ignatieff, M. (1987). An Interview with Bruce Chatwin. UK: Granta.

Meanor, P. (1996). Bruce Chatwin. New York: Twayne's English Authors Series.

Murray, N. (1993). Bruce Chatwin. Mid Glamorgan (Wales): Seren Books.

Rushdie, S. (1992). Imaginary Homelands. NY: Granta Books.

Shakespeare, N. (1999). Bruce Chatwin. London: Harvill Press.

\section{BY-NC-ND}

